\title{
多功能固态量子存储器取得新突破
}

周宗权，李传锋*，郭光灿

中国科学技术大学, 中国科学院量子信息重点实验室, 合肥 230026

*联系人, E-mail: cfli@ustc.edu.cn

\section{New breakthrough of multifunctional solid state quantum memory}

\section{Zongquan Zhou, Chuanfeng $\mathrm{Li}^{*}$ \& Guangcan Guo}

\author{
CAS Key Laboratory of Quantum Information, University of Science and Technology of China, Hefei 230026, China \\ * Corresponding author, E-mail: cfli@ustc.edu.cn \\ doi: 10.1360/TB-2019-0245
}

由于不可克服的光纤传输损耗, 目前地面上的安全量 子通信被限制在百千米量级. 如何实现千千米量级的长程 量子通信是量子通信领域面临的重要难题之一. 目前物理 上的解决方案有两种: 第一种是基于量子中继 ${ }^{[1]}$, 利用短 程的量子纠缠和量子存储器实现长程的量子纠缠, 再利用 量子隐形传态等实现量子通信; 第二种是直接把量子信息 存储到寿命超长的量子存储器 ${ }^{[2]}$ 中, 利用经典运输手段, 比如飞机、卡车和高铁等, 进行长途运输. 显然量子存储 器是实现这两种长程量子通信方案的关键器件. 此外, 量 子计算领域也需要高速的量子存储器(寄存器)来实现高速 量子信息处理和线性光学量子计算等. 在此背景下, 我国 于2014年启动了国家重大科研仪器设备研制专项“多功能 固态量子存储器”, 项目由郭光灿院士负责, 中国科学技 术大学承担. 经过 5 年艰苦努力, 本项目于 2019 年6月正式 通过国家自然科学基金委员会验收, 研制的多功能固态量 子存储器各项指标均达到或超过立项时的设计要求.

常见的量子存储器有热原子、冷原子、腔内单原子和 稀土掺杂晶体. 稀土掺杂晶体具有性能稳定、易于扩展和 加工等优点, 并且稀土离子的 $4 \mathrm{f}$ 价电子被外层 $5 \mathrm{~s}$, $5 \mathrm{p}$ 等电子 屏蔽, 相干性保持得很好, 因此本项目选择利用稀土掺杂 晶体研制多功能固态量子存储器. 利用原子频率梳技术 ${ }^{[3]}$ 实现存储过程, 这个技术与光学频率梳有些类似. 稀土离 子的本征线宽只有 $10 \mathrm{kHz}$ 量级, 但是掺杂到晶体中后线宽 会被晶体场非均匀展宽到几百 $\mathrm{MHz}$, 甚至 THz. 利用光学 烧孔的办法, 可以把稀土离子的下能级处理成等间隔的频 率梳状结构. 当输人的光子脉冲被稀土离子吸收后, 由于 自聚焦效应, 隔一段时间后光子脉冲会被释放出来, 存储 时间反比于频率梳间距. 由于频率梳间距不可能小于稀土 离子的本征线宽 (10 kHz量级), 所以存储时间有限. 为了延
长存储时间, 需要另外一个下能级(通常寿命会很长, 目前 的纪录是 $6 \mathrm{~h}$ ). 当光子脉冲被吸收后, 利用一个 $\pi$ 脉冲把稀 土离子从上能级转到这个下能级上, 等到需要的时候再用 另一个 $\pi$ 脉冲把稀土离子转移到上能级, 然后继续自聚焦 演化放出光子脉冲. 这样一个完整的原子频率梳存储过程, 存储时间取决于下能级的寿命, 原则上可以达到小时量级. 稀土元素共有 17 种, 原则上每种稀土离子都可以用来做量 子存储. 常见的掺杂晶体有钒酸钇、硅酸钇等. 稀土掺杂 晶体通常需要放在 $3 \mathrm{~K}$ 以下的低温下, $0.1 \mathrm{~T}$ 量级的磁场中 工作. 根据价电子的配对情况, 稀土离子可以分为两种: 一种是Kramers离子, 有未配对电子. 如 $\mathrm{Er}, \mathrm{Nd}, \mathrm{Yb}$ 离子, 具有电子自旋, 一般具有较宽的存储带宽, 但是存储寿命 受限于电子自旋与外场涨落的耦合. 另一种是Non- Kramers离子, 无未配对电子. 如 $E u, P r, T m$ 离子, 只有核自旋, 带宽一般受限于精细结构繴裂, 为 $10 \mathrm{MHz}$ 量级. 核自旋与 环境耦合弱，存储寿命较长. 根据稀土离子的分类，研制 的多功能固态量子存储器包括长寿命固态量子存储器和 宽带固态量子存储器两部分(图1). 仪器结构主要包括围绕 存储介质的光学系统、电学系统以及配套测试系统. 项目 组重点突破了宽带量子存储、高保真量子存储及长寿命量 子存储等关键技术. 经过持续优化改进, 所研制的多功能 固态量子存储器存储寿命达 $2.067 \mathrm{~ms}$ (此前国际同行达到 $1.5 \mathrm{~ms})$, 慢光传输速度达 $700 \mathrm{~m} / \mathrm{s}$, 存储带宽达 $1046 \mathrm{MHz}$, 存储保真度达 $99.8 \%$ (此前国际同行达到 $97.5 \%$ ), 存储模式 数达 100 个(此前国际同行达到 64 个), 匹配的光子对亮度达 到3025光子对 $\mathrm{s}^{-1}(100 \mathrm{MHz})^{-1}$. 各项技术指标均达到该领 域国际一流水平.

依托该重大仪器, 项目组开展了一系列有国际影响力 的实验研究：(1) 首次实现量子点发射的确定性单光子固 

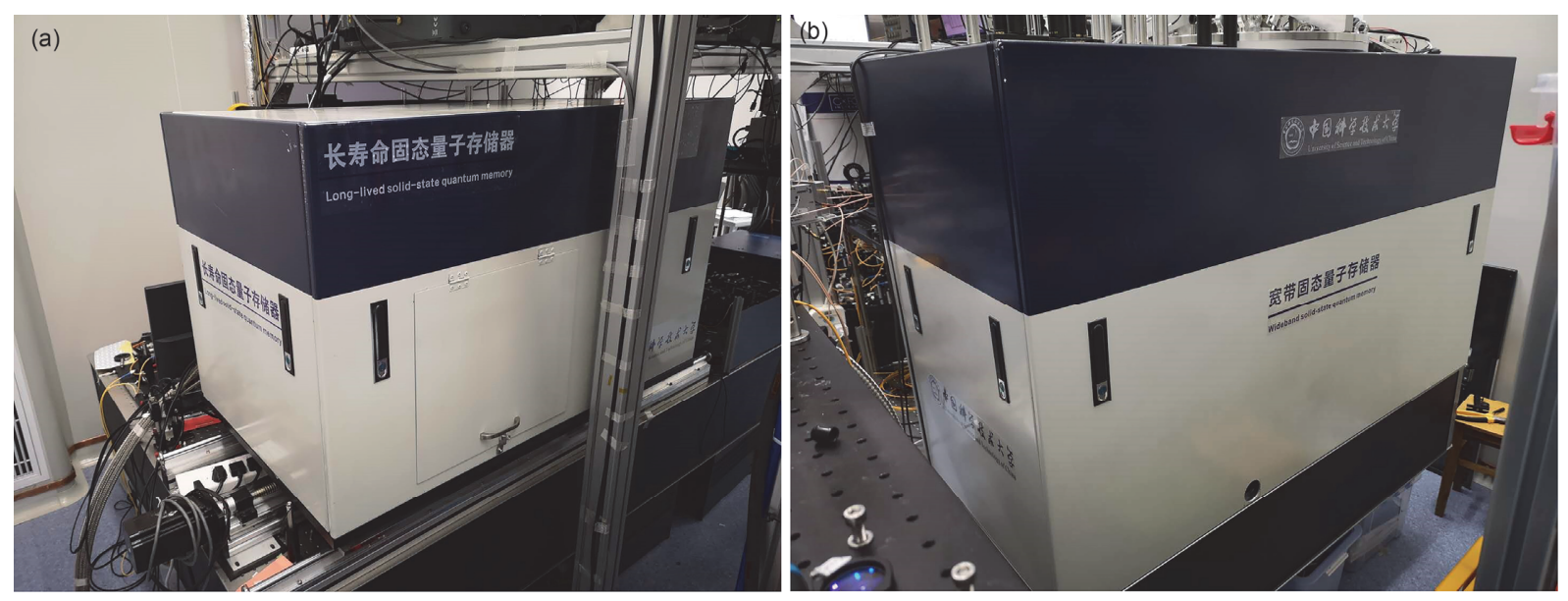

图 1 (网络版彩色)多功能固态量子存储器外观图. (a) 长寿命固态量子存储器. 存储寿命达 $2.067 \mathrm{~ms}$, 慢光传输速度达 $700 \mathrm{~m} / \mathrm{s}$. (b) 宽带固态 量子存储器. 存储带宽达 $1046 \mathrm{MHz}$, 存储保真度达 $99.8 \%$, 存储模式数达 100 个, 匹配的光子对亮度达到 3025 光子对 s s $^{-1}(100 \mathrm{MHz})^{-1}$

Figure 1 (Color online) Outside view of multi-functional solid state quantum memory. (a) Long-lived solid-state quantum memory. The storage time is $2.067 \mathrm{~ms}$, the slowlight propagation speed is $700 \mathrm{~m} / \mathrm{s}$. (b) Wideband solid-state quantum memory. The storage bandwidth is $1046 \mathrm{MHz}$, the fidelity is $99.8 \%$, the mode number is 100 , and the brightness of matched photon pair is 3025 pairs s$^{-1}(100 \mathrm{MHz})^{-1}$

态量子存储器, 构建了基于两种异种固态系统的雉形量子 ${ }^{\text {网络 }}{ }^{[4]}$; (2) 首次实现轨道角动量量子态以及高维纠缠态 的固态量子存储 ${ }^{[5]} ;(3)$ 首次实现时间、空间、频率3个自 由度并行复用的多功能固态量子存储, 并演示量子信息在 不同模式间的变换 ${ }^{[6]}$; (4) 首次在毫米尺寸实现宏观实在 性(Leggett-Garg不等式)的严格检验, 为解决薛定谔猫佯谬
问题提供了新思路 ${ }^{[7]}$.

该多功能固态量子存储器的成功研制，对于可扩展大 尺度量子网络构建提供了有力技术支撑，其慢光功能也为 研究量子力学与相对论的相容性等重要基础物理问题打 下重要基础. 在此仪器基础上, 下一步将可以实现量子中 继节点和量子加密优盘, 逐步推进长程量子通信的研究.

\section{参考文献}

1 Sangouard N, Simon C, de Riedmatten H, et al. Quantum repeaters based on atomic ensembles and linear optics. Rev Mod Phys, 2011, 83: $33-80$

2 Zhong M, Hedges M P, Ahlefeldt R L, et al. Optically addressable nuclear spins in a solid with a six-hour coherence time. Nature, 2015, 517: $177-180$

3 de Riedmatten H, Afzelius M A, Staudt M U, et al. A solid-state light-matter interface at the single-photon level. Nature, 2008, 456: $773-777$

4 Tang J S, Zhou Z Q, Wang Y T, et al. Storage of multiple single-photon pulses emitted from a quantum dot in a solid-state quantum memory. Nat Commun, 2015, 6: 8652

5 Zhou Z Q, Hua Y L, Liu X, et al. Quantum storage of three-dimensional orbital-angular-momentum entanglement in a crystal. Phys Rev Lett, 2015, 115: 070502

6 Yang T S, Zhou Z Q, Hua Y L, et al. Multiplexed storage and real-time manipulation based on a multiple degree-of-freedom quantum memory. Nat Commun, 2018, 9: 3407

7 Zhou Z Q, Huelga S F, Li C F, et al. Experimental detection of quantum coherent evolution through the violation of Leggett-Garg-type inequalities. Phys Rev Lett, 2015, 115: 113002 